\title{
Altered Fingerprint Matching Using Ridge Texture and Frequency in the Unaltered Region
}

\author{
T.R. Anoop and M.G. Mini
}

\begin{abstract}
Fingerprint alteration is the process of changing the regularly spaced ridge structure by mechanical or chemical means to hide the identity from Automated Fingerprint Identification System. This paper presents a method for altered fingerprint matching using texture and ridge frequency in the unaltered region. Wavelet transform is used to create feature vectors from ridge frequency and ridge texture. Matching score obtained for both texture and ridge frequency is fused together to obtain final score. Results of one to many matching between synthetically altered fingerprints and its unaltered mates shows that this method is suitable for altered fingerprint matching.
\end{abstract}

Keywords--- Altered Fingerprints Matching, Ridge Texture, Ridge Frequency, Wavelet Transform

\section{INTRODUCTION}

$\mathrm{F}$ INGREPRINTS are the widely used biometrics for personal identification system since feature of the fingerprints(FP) are unique for each human being. Fake and altered fingerprints are some of the threats to personal identification system. Fake FPs are made from latex and are used by the criminals to break the automatic personal identification systems to enter into restricted areas. Fingerprint alteration is the process of making the regular ridge structure irregular to hide the identity from the personal identification system or from the watch list of fingerprints owned by the security. Altered FP matching is essential to identify the criminals by matching it against its unaltered mates in the watch list. The fingerprint alteration is divided by subjective assessment into three types; obliteration, distortion and imitation [1],[2]. Fingerprints are altered by cutting, abrasion with blades, poring chemicals, burning and surgery. Obliteration is again divided into scar and mutilation. Distorted type of alteration is formed by transplantation of ridge structure with other portions of the same fingerprints by surgery. Imitation type is obtained by replacing large area of fingertip with portion of other fingerprints, palm prints and leg prints by plastic surgery.

Matching of imitation and distortion type of alteration is not possible since it contains transplanted ridge structure. Separation of altered region from the unaltered region and restoration of altered region is also difficult. Matching of ' $Z$ ' cut type distortion can be performed since restoration may be

T.R. Anoop, Research Scholar, Electronics Department, Model Engineering College, Kochi, India.E-mail:anooptr234@gmail.com

M.G. Mini, Associate Professor, Electronics Department, Model Engineering College, Kochi, India.E-mail:mininair@mec.ac.in possible. Soweon Yoon and Anil K. Jain have performed the matching of ' $Z$ ' cut type of distortion by the restoration of minutiae distribution in the altered region [3].

Fingerprint recognition is basically divided into three types [4]. They are correlation based, minutiae based and nonminutiae features based techniques. In correlation based techniques, two FP images are superimposed and the correlation between corresponding pixels is computed for different alignments [5],[6],[7],[8],[9]. Ridge endings and bifurcation known as minutiae features is unique for each FP. Minutiae-based matching consists of finding the alignment between the template and the input minutiae feature sets that result in the maximum number of minutiae pairings[10],[11], [12],[1] [14],[15]. In non minutiae based matching, features of the ridge pattern like local orientation, frequency, ridge shape and texture information and number, type and position of singularities are used [16],[17],[18],[19]. Some of the researchers used non-minutiae based features along with minutiae feature to improve the matching performance [20],[21],[22]. They computed the matching score separately for minutiae and non-minutiae features and combined to get the final matching score.

The proposed method belongs to texture feature based techniques and uses the ridge texture and ridge frequency in the unaltered region of altered FP. This method starts with the selection of unaltered region from the altered FP and similar region of interest from the unaltered mate. Wavelet transform techniques are applied on the ridge frequency and ridge texture images found from selected region to generate feature vectors. Final matching score is obtained by fusing the Euclidian distance of feature vectors of ridge frequency and ridge texture.

Remaining part of the paper is organized as follows. Section II explains ridge frequency extraction. Section III and IV gives the wavelet decomposition and proposed method respectively. Section V gives the results and discussion and conclusion is given in VI.

\section{RIDGE FREQUENCY EXTRACTION}

The local ridge frequency (or density) $f_{x y}$ at point $[x, y]$ is the number of ridges per unit length along a hypothetical segment centred at $[x, y]$ and orthogonal to the local ridge orientation. A frequency image $\mathrm{F}$, analogous to the orientation image $\mathrm{D}$, can be defined if the frequency is estimated at discrete positions and arranged into a matrix. The local ridge frequency varies across different fingers, and may also vary across different regions of the same fingerprint [4]. The 
method proposed by Hong, Wan, and Jain [23] is used in this

paper to estimate ridge frequency.

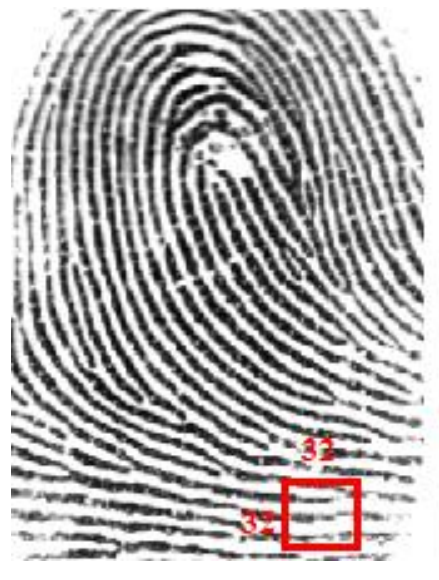

(a)

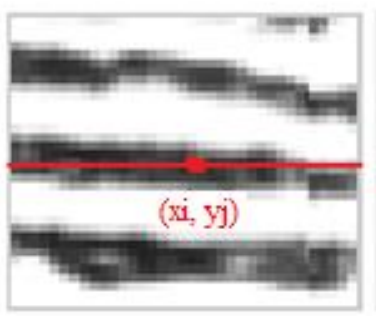

(b)

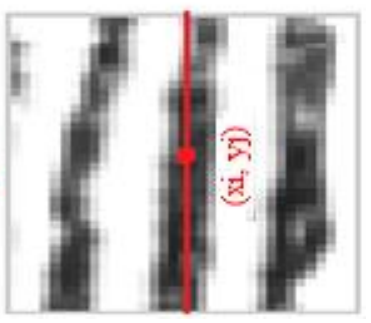

(c)

Figure 1: (a) 32X32 Window is Marked with Rectangle, (b) 32X32 Window with Center $\left(\mathrm{x}_{\mathrm{i}}, \mathrm{y}_{\mathrm{j}}\right)$, (c) Rotated

Window with Ridges Points in the Vertical Direction

In order to find the frequency $f_{i j}$ at a point $\left(x_{i}, y_{j}\right)$, a rectangular window of size $32 \times 32$ centered at $\left(x_{i}, y_{j}\right)$ is considered. Then the window is rotated in such a way that the ridges point in the vertical direction. This is shown in figure 1 . After the rotation, the gray levels in the each column are accumulated to obtain $\mathrm{x}$-signature. Then $\mathrm{f}_{\mathrm{ij}}$ is determined as the inverse of the average distance between two consecutive peaks of the $x$-signature. This is shown in figure 2. Thus $f_{i j}$ at $\left(\mathrm{x}_{\mathrm{i}}, \mathrm{y}_{\mathrm{j}}\right)$ is given by

$$
\mathrm{f}_{\mathrm{ij}}=4 /(\mathrm{s} 1+\mathrm{s} 2+\mathrm{s} 3+\mathrm{s} 4)
$$

Figure 3 shows the ridge frequency obtained by the above method.

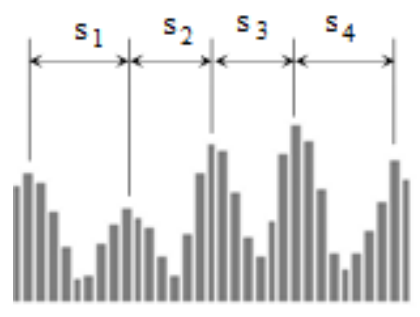

Figure 2: x-signature

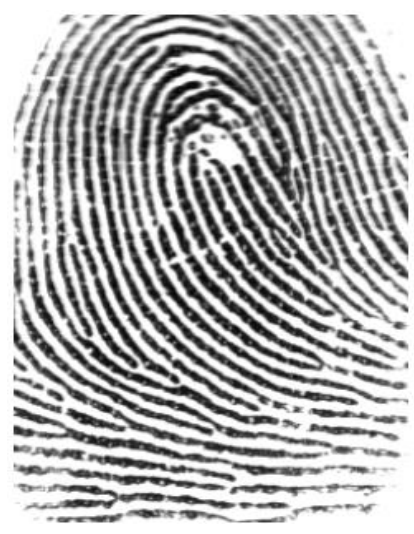

Figure 3: (a) Fingerprint,

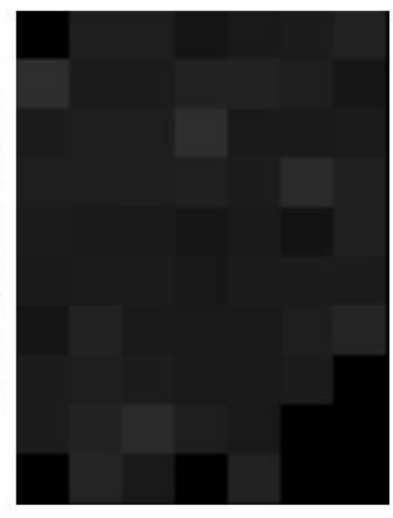

(b) Ridge Frequency Image

\section{WAVELET DECOMPOSITION}

Wavelet transform provides provision for multi resolution analysis [24], [25], [26], [27]. In the 2D case, the scaling and wavelet coefficients are computed using a 2D filter bank composed of low pass and high pass filters and decimators. At each scale, the 2D function is decomposed into an approximation image $a_{j}$ of a low pass band and three detail images $d_{j}{ }^{H}, d_{j}{ }^{V}$ and $d_{j}{ }^{D}$ corresponding to a horizontal high pass band, a vertical high pass and diagonal high pass band respectively as given below.

$$
\begin{aligned}
& w_{\phi}\left(j_{0}, m, n\right)=\frac{1}{\sqrt{M N}} \sum_{x=0}^{M-1} \sum_{y=0}^{N-1} f(x, y) \cdot \phi_{j_{0}, m, n}(x, y) \\
& w_{\psi}^{i}(j, m, n)=\frac{1}{\sqrt{M N}} \sum_{x=0}^{M-1} \sum_{y=0}^{N-1} f(x, y) \cdot \psi_{j, m, n}^{i}(x, y)
\end{aligned}
$$

Where $\mathrm{i}=\{\mathrm{H}, \mathrm{V}, \mathrm{D}\}, \mathrm{j} 0$ is an arbitrary starting scale, $\mathrm{w}_{\phi}(\mathrm{j} 0, \mathrm{~m}, \mathrm{n})$ define the approximation coefficients and $w_{\psi}^{i}(j, m, n)$ defines the detailed coefficients in horizontal, vertical and diagonal directions.

\section{PROPOSED METHOD}

After finding the region of interest from a fingerprint, ridge frequency image is formed. Both the ridge frequency image and selected ROI are decomposed using wavelet transform and matching score is obtained in the form of Euclidian distance. Fusion of matching score of ridge frequency image and ridge texture is performed to obtain final matching score. This is explained in following sub sections.

\section{A. Selection of Region of Interest (ROI)}

Altered fingerprint matching can be done in two ways. One needs the restoration of the altered region and other needs the features in the unaltered region. These two ways of matching fails when the whole area of the fingerprint is altered. Region of interest (ROI) is the unaltered region 
selected from the altered FP. Similar regions are also selected from the entire normal fingerprint in the database consisting of unaltered mates. Figure 1 shows the synthetically altered FP and its unaltered mate along with other normal fingerprints. Unaltered region belongs to bottom of the altered FP and hence similar region with same size is selected from normal fingerprints as shown in figure 4(b), (c) and (d). If the alteration is at the bottom region, then the top end region of the fingerprint is selected. See figure 5 .

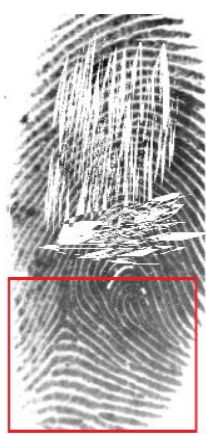

(a)

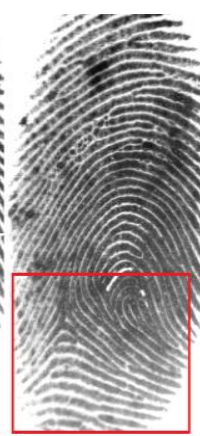

(b)

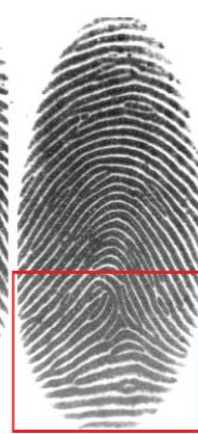

(c)

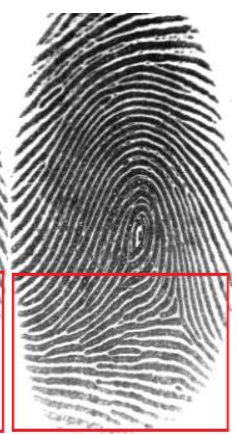

(d)
Figure 4: Region of interest marked as Rectangle in Altered and Unaltered Fingerprints. (a) Synthetically Altered FP, (b) Unaltered Mate, (c) \& (d) Unaltered FP in the Database
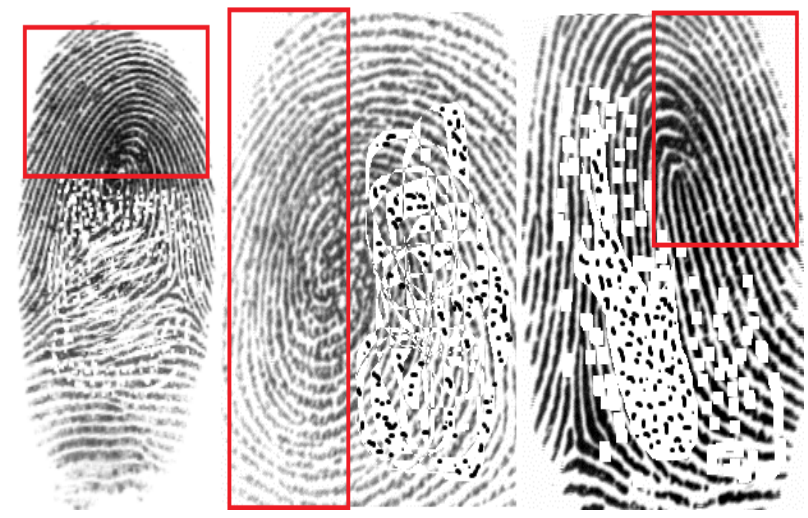

Figure 5: ROI Selected from Altered FP

\section{B. Formulation of Matching Score from Ridge Frequency Image}

Ridge frequency images for both altered FP and normal FP are obtained as explained in section II. These frequency images are decomposed up to 4 levels using Haar wavelet and the mean of approximation coefficients at the $4^{\text {th }}$ level and detailed coefficients at all the levels are found. The Matching score is obtained by finding the Euclidian distance between these two feature vectors as follows.

$E R F=\sqrt{\sum_{N=1}^{13}\left(\left(m R F_{-} A F P(N)-m F P_{-} N F P(N)\right)^{2}\right.}$

where ERF is Euclidian distance for ridge frequency, mRF_AFP(N) and mFP_NFP(N) is the mean of the coefficients of ridge frequency image of altered FP and normal FP respectively. $\mathrm{N}$ varies from 1 to 13 .

\section{Formulation of Matching Score from Ridge Texture}

The ROI from altered and normal FP is decomposed to fourth level by Haar wavelet to find out ridge texture feature vectors. Procedure followed is same as explained for ridge frequency.

$E R T=\sqrt{\sum_{N=1}^{13}\left(\left(m R T_{-} A F P(N)-m F N_{-} N F P(N)\right)^{2}\right.}$

where ERT is Euclidian distance for ridge texture, mRF_AFP(N) and mFP_NFP(N) is the mean of the coefficients of ridge texture image of altered FP and normal FP respectively.

\section{Formulation of Final Matching Score}

Matching scores obtained by comparing ridge frequency features and ridge textures are combined to get a single matching score. In order to do this, we have used weighted sum rule [18]. Final matching score EFS is generated as follows.

$$
\mathrm{EFS}=\alpha . \mathrm{ERF}+(1-\alpha) \mathrm{ERT}
$$

Where $\alpha$ varies between 0 and 1 . The value of ERT is very high as compared to ERF. Thus $\alpha$ is taken as 0.98 to normalize ERT to a value in between 0 and 1 .

\section{RESUlts AND DisCUSSION}

An altered FP database consisting of 70 synthetically altered fingerprints is used for the experiments. Normal fingerprints obtained from FVC 2000 and FVC 2004 database is used for creating synthetically altered fingerprints. Each group in this database consists of 8 impressions of 10 fingers and is captured by optical and capacitive sensors. The resolution of each fingerprint is 500dpi. Synthetically altered obliteration of different area is created by making scar and scratches in the image.

Each altered FP is matched with the entire fingerprint in the normal FP database (one to many matching). A matching is declared as successful, if value of EFS less than or equal to a threshold ' $t$ '. Table 1 gives the results of matching in terms of Genuine Acceptance Rate (GAR) and False Acceptance Rate (FAR) for the selected threshold. GAR gives the successful matching of altered FP and FAR gives the false match rate.

Table 1: Results of Matching. GAR and FAR in \%

\begin{tabular}{|c|c|c|}
\hline No. of Altered FP & GAR in \% & FAR in \% \\
\hline Altered FP(70) & 84.28 & 12.85 \\
\hline
\end{tabular}

ROC curve is plotted for the matching score obtained for this method and is shown in figure.6. 


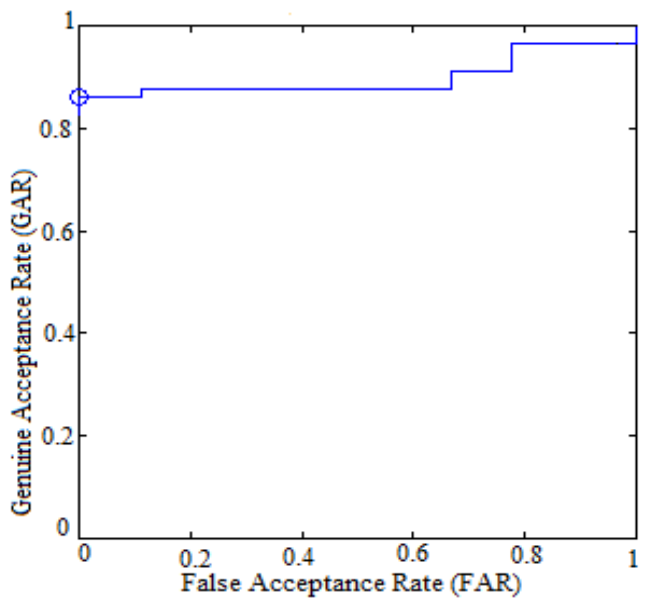

Figure 6: ROC Curve for Matching Score

\section{CONCLUSION AND FUTURE ENHANCEMENT}

This paper presents a method to match obliteration type of altered fingerprint with its unaltered mates. Ridge frequency and ridge texture in the unaltered region of the altered FP is used for matching. Features are extracted by wavelet decomposition of ridge frequency and ridge texture. Matching score from ridge texture and ridge frequency is combined to get final matching score. Results show that this method is suitable for altered FP matching. Comparison of the result is not possible since the state of the art methods for altered FP matching is not available.

\section{REFERENCES}

[1] J. Feng, A.K. Jain, and A. Ross, Detecting Altered Fingerprints, Proc. 20th Int'l Conf. Pattern Recognition, pp. 1622-1625, Aug. 2010.

[2] Soweon Yoon, Jianjiang Feng, and Anil K. Jain, Altered Fingerprints: Analysis and Detection'. IEEE transactions on pattern analysis and machine intelligence, Vol. 34, No. 3, March 2012

[3] Soweon Yoon, Qijun Zhao, and Anil K. Jain, "On Matching Altered Fingerprints" International conference on biometrics, New Delhi, India, March 2012.

[4] D. Maltoni, D. Maio, A.K. Jain, and S. Prabhakar, Handbook of Fingerprint Recognition, second ed. SpringerVerlag, 2009

[5] A. M. Bazen, G. T. B. Verwaaijen, S. H. Gerez, L. P. J. Veelenturf, and B. J. van der Zwaag. A CorrelationBased Fingerprint Verification System. In Proceedings of Workshop on Circuits Systems and Signal Processing, pages 205-213, 2000.

[6] Kumar, Savvides M., Xie C., Venkataramani K., Thornton J. and Mahalanobis A., "Biometric verification with correlation filters," Applied Optics, vol. 43, no. 2, pp. 391-402, 2004.

[7] Nandakumar, K., Jain, A.: Local correlation-based fingerprint matching. Proc. of Indian Conference on Computer Vision, Graphics and Image Processing pp. 503-508 (2004)

[8] T. Hatano, T. Adachi, S. Shigematsu, H. Morimura, S. Onishi, Y. Okazaki, H. Kyuragi, "A fingerprint verification algorithm using the differential matching rate", Proceedings of the 16th International Conference on Pattern Recognition, pp. 799-802, 2002.

[9] A. Cavusoglu, S. Gorgunoglu, "A robust correlation based fingerprint matching algorithm for verification”, J. Appl. Sci.,pp.3286-3291, 2007.

[10] Johannesen F.R., Raaschou S., Larsen O.V. and Jurgensen P., "Using Weighted Minutiae for Fingerprint Identification," in Proc. Advances in Structural and Syntactical Pattern Recognition, pp. 289-299, 1996.

[11] Jie Y., Fang Y.Y., Renjie Z. and Qifa S., "Fingerprint minutiae matching algorithm for real time system," Pattern Recognition, vol. 39, no. 1, pp. 143-146, 2006.

[12] X. Luo, J. Tian, Y. Wu, A minutia matching algorithm in fingerprint verification, Proceedings of the International Conference on Pattern Recognition (15th), vol. 4, 2000, pp. 833-836
[13] Jea and Govindaraju (2005). Jea T.Y. and Govindaraju V., "A minutiabased partial fingerprint recognition system," Pattern Recognition, vol. 38, no. 10, pp. 1672-1684, 2005.

[14] Haiyong Chen, Hongwei Sun, Kwok-Yan Lam, A fast and elastic fingerprint matching algorithm using minutiae-centered circular regions, Intl. Conf. on Emerging Security Information, Systems and Technologies, IEEE, Oct. 14-20, 2007.

[15] Neeta Nain , Deepak B M, Dinesh Kumar, Manisha Baswal, and Biju Gautham "Optimized Minutiae-Based Fingerprint Matching" Proceedings of the World Congress on Engineering 2008 Vol I WCE 2008, July 2 - 4, 2008, London, U.K

[16] Jain A.K., Prabhakar S., Hong L. and Pankanti S., "FingerCode: A Filterbank for Fingerprint Representation and Matching," in Proc. Conf. Computer Vision and Pattern Recognition,nvol. 2, pp. 187-193, 1999

[17] Jain A.K., Prabhakar S., Hong L. and Pankanti S., "Filterbank-based fingerprint matching," IEEE Transactions on Image Processing, vol. 9, pp. 846-859, 2000.

[18] Zin Mar Win and Myint Myint Sein, Robust Fingerprint Recognition System using Orientation and Texture features 2nd International Conference on Computer Science and Information Technology (ICCSIT'2012) Singapore April 28-29, 2012

[19] Jayant V. Kulkarni, Bhushan D. Patil, Raghunath S. Holambe "Orientation feature for fingerprint matching", March 2006 Pattern Recognition 39 (2006) 1551 - 1554

[20] Marius Tico, ,and Pauli Kuosmanen "Fingerprint Matching Using an Orientation-Based Minutia Descriptor" IEEE Transaction on is and Pattern analysis and Machine Intelligence, Vol.25. No.8, August 2003.

[21] Fanglin Chen, Jie Zhou, and Chunyu Yang "Reconstructing Orientation Field From Fingerprint Minutiae to Improve Minutiae-Matching Accuracy" .IEEE transaction on image processing, Vol.18, No.7, July 2009.

[22] Jin Qi, Suzhen Yang, Yangsheng Wang Fingerprint matching combining the global orientation field with minutia Pattern Recognition Letters 26 (2005) 2424-2430 17 June 2005

[23] L.Hong, Y.Wan and A.K.Jain, "Fingerprint image Enhancement: Algorithm and Performance Evaluation," IEEE Transaction on Pattern Analysis and Machine Intelligence, Vol. 20, no. 8, pp. 777-789, Aug. 1998.

[24] Chebira et al. (2007). Chebira A, Coelho L.P., Sandryhaila A., Lin S., Jenkinson W.G., MacSleyne J., Hoffman C., Cuadra P., Jackson C., Puschel M. and Kovacevic J., "An Adaptive Multiresolution Approach to Fingerprint Recognition," in Proc. Int. Conf. on Image Processing, vol. 1, pp. 457-460, 2007.

[25] Nanni and Lumini (2007). Nanni L. and Lumini A., "A hybrid waveletbased fingerprint matcher," Pattern Recognition, vol. 40, no. 11, pp. 3146-3151, 2007.

[26] Huang and Aviyente (2004a). Huang K. and Aviyente S., "Choosing Best Basis in Wavelet Packets for Fingerprint Matching," in Proc. Int. Conf. on Image Processing, vol. 2, pp. 1249-1252, 2004

[27] Jawerth and W. Sweldens, "An overview of Wavelet based multiresolution analysis," SIAM Review, vol. 36, no. 3, pp.377 412,September1994

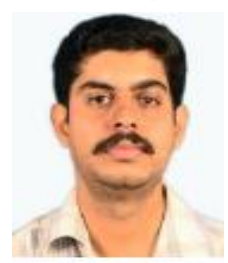

Anoop.T.R. graduated in electronics and communication engineering and received M.E in VLSI design from Anna University, Chennai in 2008. Presently he is doing $\mathrm{PhD}$ in image processing in Model Engineering college, research centre of Cochin University of Science and Technology, Kochi, India. He presented VLSI and image processing based papers in national and international conferences and journals. His areas of interest include VLSI design and image

processing.

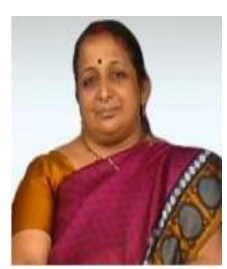

Dr.Mini M.G graduated in Electronics and Communication engineering from University of Kerala in 1988. She received M.Tech in Digital electronics in 1994 and $\mathrm{PhD}$ in image processing in 2005 from Cochin University of Science and Technology, Kochi, Kerala, India. Presently she is working as Associate Professor and Academic Head, Model Engineering College, Kochi, India. Her areas of interest include Digital Image processing, ASIC Design, Digital system design 